\title{
Structural Modulation of Fluorescent Rhodamine-Based Dysprosium(III) Single-Molecule Magnets
}

\author{
Mei-Jiao Liu, ${ }^{\dagger}$ Shu-Qi Wu, ${ }^{\S}$ Jia-Xin Li, $\$$ Yi-Quan Zhang, ${ }^{*} \pitchfork$ Osamu Sato and
}

\author{
Hui-Zhong Kou*†
}

$\dagger$ Department of Chemistry, Tsinghua University, Beijing, 100084, P. R. China. E-mail: kouhz@mail.tsinghua.edu.cn

$\ddagger$ Jiangsu Key Laboratory for NSLSCS, School of Physical Science and Technology, Nanjing Normal University, Nanjing, 210023, P. R. China. E-mail: zhangyiquan@njnu.edu.cn

$\S$ Institute for Materials Chemistry and Engineering \& IRCCS, Kyushu University, 744 Motooka, Nishi-ku, Fukuoka 819-0395, Japan

Table S1. Data structural and refinement parameters for complexes $\mathbf{1}$ and $\mathbf{2 .}$

\begin{tabular}{|lll|}
\hline Complexes & $\mathbf{1}$ & $\mathbf{2}$ \\
\hline Formula & $\mathrm{C}_{64.5} \mathrm{H}_{71} \mathrm{Cl}_{3} \mathrm{DyN}_{10} \mathrm{O}_{20}$ & $\mathrm{C}_{37} \mathrm{H}_{50} \mathrm{Cl}_{3} \mathrm{DyN}_{6} \mathrm{O}_{21}$ \\
Formula weight & 1575.16 & 1183.68 \\
$T / \mathrm{K}$ & 100 & 100
\end{tabular}




\begin{tabular}{|lll|} 
Crystal system & trigonal & monoclinic \\
Space group & $P \overline{1}$ & $P 2_{1} / \mathrm{n}$ \\
$a / \AA$ & $14.0558(5)$ & $16.7686(3)$ \\
$b / \AA$ & $14.4902(4)$ & $14.3325(2)$ \\
$c / \AA$ & $19.5669(5)$ & $20.4434(4)$ \\
$\alpha /{ }^{\circ}$ & $88.515(2)$ & 90 \\
$\beta /{ }^{\circ}$ & $89.849(2)$ & $108.926(2)$ \\
$\gamma /{ }^{\circ}$ & $62.965(3)$ & 90 \\
$V / \AA^{3}$ & $3548.36(19)$ & $4647.66(16)$ \\
$Z$ & 2 & 4 \\
$\rho_{\text {calc }} / \mathrm{g}^{-\mathrm{cm}^{-3}}$ & 1.474 & 1.692 \\
$\mu / \mathrm{mm}^{-1}$ & 7.332 & 10.948 \\
$\mathrm{~F}(000)$ & 1610 & 2396 \\
Radiation & $\mathrm{CuK \alpha}$ & $\mathrm{CuK} \alpha$ \\
Data/restraints/parameters & $13393 / 60 / 956$ & $8775 / 58 / 693$ \\
$\mathrm{GOF}$ on $F^{2}$ & 1.036 & 1.019 \\
$R_{\text {int }}$ & 0.0489 & 0.0329 \\
$R 1, w R 2[I>2 \sigma(I)]$ & $0.0703,0.1906$ & $0.0505,0.1261$ \\
$R 1, w R 2($ all data $)$ & $0.0772,0.1894$ & $0.0548,0.1298$ \\
\hline
\end{tabular}

Table S2. Selected bond lengths ( $\AA$ ) for complexes $\mathbf{1}$ and $\mathbf{2}$.

\begin{tabular}{|ll|ll|}
\hline \multicolumn{2}{|c|}{$\mathbf{1}$} & \multicolumn{2}{|c|}{} \\
\hline Dy-O1 & $2.172(4)$ & Dy-O1 & $2.175(3)$ \\
Dy-O2 & $2.380(3)$ & Dy-O2 & $2.379(3)$ \\
Dy-O3 & $2.381(4)$ & Dy-O1W & $2.383(3)$ \\
Dy-O4 & $2.414(4)$ & Dy-O2W & $2.391(3)$ \\
Dy-N1 & $2.528(5)$ & Dy-O3W & $2.350(4)$ \\
Dy-N2 & $2.578(5)$ & Dy-O4W & $2.408(4)$ \\
Dy-N3 & $2.566(4)$ & Dy-N1 & $2.524(4)$ \\
Dy-N4 & $2.632(5)$ & Dy-N2 & $2.519(4)$ \\
Dy-N5 & $2.531(5)$ & & \\
\hline
\end{tabular}


Table S3. The results of coordination geometric configurations evaluated by SHAPE software for complexes $\mathbf{1}$ and 2.

\begin{tabular}{|c|c|c|c|c|c|}
\hline Symmetry & Shape & 1 & Symmetry & Shape & 2 \\
\hline $\mathrm{D}_{9 \mathrm{~h}}$ & Enneagon & 32.420 & $\mathrm{D}_{8 \mathrm{~h}}$ & Octagon & 29.728 \\
\hline $\mathrm{C}_{8 \mathrm{v}}$ & Octagonal pyramid & 22.489 & $\mathrm{C}_{7 \mathrm{v}}$ & Heptagonal pyramid & 23.021 \\
\hline $\mathrm{D}_{7 \mathrm{~h}}$ & Heptagonal bipyramd & 16.413 & $\mathrm{D}_{6 \mathrm{~h}}$ & Hexagonal bipyramid & 15.074 \\
\hline $\mathrm{C}_{3 \mathrm{v}}$ & $\begin{array}{l}\text { Johnson triangular } \\
\text { cupola J3 }\end{array}$ & 12.823 & $\mathrm{O}_{\mathrm{h}}$ & Cube & 11.472 \\
\hline $\mathrm{C}_{4 \mathrm{v}}$ & Capped cube J8 & 8.653 & $\mathrm{D}_{4 \mathrm{~d}}$ & Square antiprism & 1.564 \\
\hline $\mathrm{C}_{4 \mathrm{v}}$ & $\begin{array}{l}\text { Spherical-relaxed } \\
\text { capped cube }\end{array}$ & 7.626 & $\mathbf{D}_{2 \mathrm{~d}}$ & $\begin{array}{l}\text { Triangular } \\
\text { dodecahedron }\end{array}$ & 1.187 \\
\hline $\mathrm{C}_{4 \mathrm{v}}$ & $\begin{array}{l}\text { Capped square } \\
\text { antiprism J10 }\end{array}$ & 2.828 & $\mathrm{D}_{2 \mathrm{~d}}$ & $\begin{array}{l}\text { Johnson } \\
\text { gyrobifastigium J26 }\end{array}$ & 12.710 \\
\hline $\mathrm{C}_{4 \mathrm{v}}$ & $\begin{array}{l}\text { Spherical capped } \\
\text { square antiprism }\end{array}$ & 1.844 & $\mathrm{D}_{3 \mathrm{~h}}$ & $\begin{array}{l}\text { Johnson elongated } \\
\text { triangular bipyramid } \\
\text { J14 }\end{array}$ & 28.544 \\
\hline $\mathrm{D}_{3 \mathrm{~h}}$ & $\begin{array}{l}\text { Tricapped trigonal } \\
\text { prism J51 }\end{array}$ & 2.079 & $\mathrm{C}_{2 \mathrm{v}}$ & $\begin{array}{l}\text { Biaugmented trigonal } \\
\text { prism J50 }\end{array}$ & 1.494 \\
\hline $\mathrm{D}_{3 \mathrm{~h}}$ & $\begin{array}{l}\text { Spherical tricapped } \\
\text { trigonal prism }\end{array}$ & 1.914 & $\mathrm{C}_{2 \mathrm{v}}$ & $\begin{array}{l}\text { Biaugmented trigonal } \\
\text { prism }\end{array}$ & 1.579 \\
\hline $\mathrm{C}_{3 \mathrm{v}}$ & $\begin{array}{l}\text { Tridiminished } \\
\text { icosahedron J63 }\end{array}$ & 1.914 & $\mathrm{D}_{2 \mathrm{~d}}$ & Snub diphenoid J84 & 2.521 \\
\hline $\mathrm{C}_{2 \mathrm{v}}$ & Hula-hoop & 10.824 & $\mathrm{~T}_{\mathrm{d}}$ & Triakis tetrahedron & 11.894 \\
\hline $\mathrm{C}_{\mathrm{s}}$ & Muffin & 1.983 & $\mathrm{D}_{3 \mathrm{~h}}$ & $\begin{array}{l}\text { Elongated trigonal } \\
\text { bipyramid }\end{array}$ & 24.410 \\
\hline
\end{tabular}


Table S4 Fitting parameter obtained from Cole-Cole for $\mathbf{1}$ in zero field.

\begin{tabular}{|c|c|c|c|c|c|c|c|c|}
\hline $\begin{array}{l}T \\
(\mathbf{K})\end{array}$ & $\begin{array}{l}\chi_{\mathrm{s}}\left(\mathrm{cm}^{3}\right. \\
\left.\mathrm{mol}^{-1}\right)\end{array}$ & $\operatorname{dev}\left(\chi_{\mathrm{s}}\right)$ & $\begin{array}{l}\chi_{\mathrm{T}}\left(\mathrm{cm}^{3} \mathrm{~mol}\right. \\
\left.{ }^{-1}\right)\end{array}$ & $\operatorname{dev}\left(\chi_{T}\right)$ & $\tau(\mathbf{s})$ & $\operatorname{dev}(\tau)$ & $\alpha$ & $\operatorname{dev}(\alpha)$ \\
\hline 2 & 0.38655 & 0.04641 & 7.82707 & 0.05398 & 0.00972 & $1.76553 \mathrm{E}-4$ & 0.29372 & 0.00836 \\
\hline 2.5 & 0.300081 & 0.03862 & 6.30141 & 0.04366 & 0.00937 & $1.72717 \mathrm{E}-4$ & 0.29432 & 0.0085 \\
\hline 3 & 0.25397 & 0.0334 & 5.23562 & 0.03664 & 0.00901 & $1.6985 \mathrm{E}-4$ & 0.29244 & 0.00874 \\
\hline 3.5 & 0.23916 & 0.028 & 4.48072 & 0.0299 & 0.0087 & $1.58511 \mathrm{E}-4$ & 0.28676 & 0.00854 \\
\hline 4 & 0.20322 & 0.02666 & 3.92676 & 0.02738 & 0.00827 & $1.60285 \mathrm{E}-4$ & 0.28897 & 0.00906 \\
\hline 4.5 & 0.18643 & 0.02297 & 3.47839 & 0.02255 & 0.0078 & $1.43555 \mathrm{E}-4$ & 0.28457 & 0.00867 \\
\hline 5 & 0.18706 & 0.02116 & 3.12596 & 0.02025 & 0.00754 & $1.4092 \mathrm{E}-4$ & 0.27866 & 0.00888 \\
\hline 6 & 0.16458 & 0.01909 & 2.59994 & 0.01688 & 0.0068 & $1.32799 \mathrm{E}-4$ & 0.27213 & 0.00935 \\
\hline 7 & 0.15004 & 0.01703 & 2.22038 & 0.0138 & 0.00606 & $1.19156 \mathrm{E}-4$ & 0.2635 & 0.00946 \\
\hline 8 & 0.14301 & 0.01589 & 1.93906 & 0.01178 & 0.00539 & $1.09419 \mathrm{E}-4$ & 0.25197 & 0.00983 \\
\hline 9 & 0.13729 & 0.01485 & 1.71722 & 0.00998 & 0.00473 & $9.77951 \mathrm{E}-5$ & 0.23905 & 0.01004 \\
\hline 10 & 0.13768 & 0.01387 & 1.53798 & 0.00844 & 0.00414 & $8.63408 \mathrm{E}-5$ & 0.22247 & 0.01019 \\
\hline 12 & 0.13752 & 0.01194 & 1.26733 & 0.00579 & 0.00307 & $6.23135 \mathrm{E}-5$ & 0.18783 & 0.00992 \\
\hline 14 & 0.13594 & 0.00964 & 1.08502 & 0.00364 & 0.00225 & $4.02355 \mathrm{E}-5$ & 0.15825 & 0.00854 \\
\hline 16 & 0.14375 & 0.00694 & 0.94438 & 0.00198 & 0.00162 & $2.27229 \mathrm{E}-5$ & 0.12486 & 0.00641 \\
\hline 18 & 0.13119 & 0.00506 & 0.83948 & $9.73225 \mathrm{E}-4$ & 0.0011 & $1.21418 \mathrm{E}-5$ & 0.10953 & 0.00434 \\
\hline 20 & 0.11706 & 0.00443 & 0.75578 & $5.08967 \mathrm{E}-4$ & $7.18013 \mathrm{E}-4$ & $7.59066 \mathrm{E}-6$ & 0.10265 & 0.00326 \\
\hline 22 & 0.11772 & 0.01203 & 0.68259 & $6.99578 \mathrm{E}-4$ & 4.39495E-4 & $1.46039 \mathrm{E}-5$ & 0.11835 & 0.00705 \\
\hline
\end{tabular}

Table S5 Fitting parameter obtained from Cole-Cole for $\mathbf{1}$ in 1000 Oe field.

\begin{tabular}{|c|c|c|c|c|c|c|c|c|}
\hline $\begin{array}{l}T \\
(\mathbf{K})\end{array}$ & $\begin{array}{l}\chi_{\mathrm{s}}\left(\mathrm{cm}^{3}\right. \\
\left.\mathbf{m o l}^{-1}\right)\end{array}$ & $\operatorname{dev}\left(\chi_{\mathrm{s}}\right)$ & $\begin{array}{l}\chi_{\mathrm{T}}\left(\mathrm{cm}^{3} \mathrm{~mol}\right. \\
\left.{ }^{-1}\right)\end{array}$ & $\operatorname{dev}\left(\chi_{T}\right)$ & $\tau(\mathbf{s})$ & $\operatorname{dev}(\tau)$ & $\alpha$ & $\operatorname{dev}(\alpha)$ \\
\hline 6 & 0.04146 & 0.00245 & 2.43712 & 0.03273 & 0.95637 & 0.02849 & 0.29017 & 0.00505 \\
\hline 8 & 0.05114 & 0.00343 & 1.6264 & 0.00941 & 0.15762 & 0.00202 & 0.21134 & 0.00536 \\
\hline 10 & 0.05396 & 0.0033 & 1.27982 & 0.00459 & 0.05011 & 4.93641E-4 & 0.17828 & 0.00489 \\
\hline 12 & 0.05446 & 0.00308 & 1.05968 & 0.00273 & 0.02021 & $1.77876 \mathrm{E}-4$ & 0.16563 & 0.0045 \\
\hline 14 & 0.06007 & 0.00242 & 0.90603 & 0.00149 & 0.00962 & $6.52115 \mathrm{E}-5$ & 0.14811 & 0.00351 \\
\hline 16 & 0.06846 & 0.00402 & 0.79527 & 0.00174 & 0.00504 & $5.59368 \mathrm{E}-5$ & 0.13329 & 0.00566 \\
\hline 18 & 0.06132 & 0.00462 & 0.70707 & 0.00128 & 0.00263 & $3.40472 \mathrm{E}-5$ & 0.14482 & 0.00575 \\
\hline 20 & 0.03382 & 0.01351 & 0.63503 & 0.00199 & 0.0013 & $5.13769 \mathrm{E}-5$ & 0.16435 & 0.01289 \\
\hline 22 & 0.09214 & 0.02112 & 0.57915 & 0.00181 & $7.84904 \mathrm{E}-4$ & $5.84842 \mathrm{E}-5$ & 0.16093 & 0.01884 \\
\hline
\end{tabular}


Table S6 Fitting parameter obtained from Cole-Cole for $\mathbf{2}$ in zero field.

\begin{tabular}{|c|c|c|c|c|c|c|c|c|}
\hline $\begin{array}{l}T \\
(\mathrm{~K})\end{array}$ & $\begin{array}{l}\chi_{\mathrm{s}}\left(\mathrm{cm}^{3}\right. \\
\left.\mathrm{mol}^{-1}\right)\end{array}$ & $\operatorname{dev}\left(\chi_{\mathrm{s}}\right)$ & $\begin{array}{l}\chi_{\mathrm{T}}\left(\mathrm{cm}^{3} \mathrm{~mol}\right. \\
\left.{ }^{-1}\right)\end{array}$ & $\operatorname{dev}\left(\chi_{\mathrm{T}}\right)$ & $\tau(\mathbf{s})$ & $\operatorname{dev}(\tau)$ & $\alpha$ & $\operatorname{dev}(\alpha)$ \\
\hline 2 & 0.12436 & 0.01766 & 8.06936 & 0.07679 & 0.26686 & 0.00651 & 0.34326 & 0.00608 \\
\hline 3 & 0.11399 & 0.01278 & 5.34044 & 0.04554 & 0.20428 & 0.00447 & 0.33156 & 0.00616 \\
\hline 4 & 0.10723 & 0.01056 & 3.97359 & 0.03275 & 0.16839 & 0.00358 & 0.32257 & 0.0065 \\
\hline 5 & 0.10017 & 0.00886 & 3.17224 & 0.02513 & 0.14822 & 0.00305 & 0.31547 & 0.00663 \\
\hline 6 & 0.092 & 0.00793 & 2.6461 & 0.02104 & 0.13459 & 0.0028 & 0.31065 & 0.00694 \\
\hline 7 & 0.08592 & 0.00724 & 2.26484 & 0.0181 & 0.12331 & 0.0026 & 0.30481 & 0.00727 \\
\hline 8 & 0.08199 & 0.00675 & 1.97844 & 0.01588 & 0.11291 & 0.0024 & 0.29537 & 0.00764 \\
\hline 9 & 0.07943 & 0.00638 & 1.75383 & 0.01403 & 0.10241 & 0.00218 & 0.28335 & 0.00801 \\
\hline 10 & 0.07801 & 0.00633 & 1.57191 & 0.01289 & 0.09163 & 0.00201 & 0.26773 & 0.00871 \\
\hline 12 & 0.07644 & 0.00565 & 1.28662 & 0.00963 & 0.06968 & 0.00143 & 0.22875 & 0.00912 \\
\hline 14 & 0.07445 & 0.00487 & 1.09317 & 0.00694 & 0.0513 & $9.31774 \mathrm{E}-4$ & 0.18881 & 0.00881 \\
\hline 16 & 0.07178 & 0.00404 & 0.94748 & 0.00482 & 0.03662 & $5.63455 \mathrm{E}-4$ & 0.15467 & 0.00795 \\
\hline 18 & 0.06789 & 0.00323 & 0.8373 & 0.00327 & 0.02572 & $3.22529 \mathrm{E}-4$ & 0.12859 & 0.00676 \\
\hline 20 & 0.06397 & 0.0029 & 0.7526 & 0.00249 & 0.01793 & $2.04427 \mathrm{E}-4$ & 0.11384 & 0.00628 \\
\hline 22 & 0.05077 & 0.00176 & 0.57675 & 0.00129 & 0.01322 & $1.06981 \mathrm{E}-4$ & 0.0951 & 0.00459 \\
\hline 24 & 0.06506 & 0.00235 & 0.62545 & 0.00141 & 0.00817 & $7.56668 \mathrm{E}-5$ & 0.09517 & 0.00521 \\
\hline 26 & 0.05185 & 0.0024 & 0.48875 & 0.0011 & 0.0052 & $5.48446 \mathrm{E}-5$ & 0.08727 & 0.00589 \\
\hline 28 & 0.06954 & 0.00233 & 0.53604 & $6.98187 \mathrm{E}-4$ & 0.00254 & $2.13717 \mathrm{E}-5$ & 0.0929 & 0.00429 \\
\hline 30 & 0.04746 & 0.00581 & 0.42293 & 7.74744E-4 & 0.00112 & $2.72842 \mathrm{E}-5$ & 0.10743 & 0.0086 \\
\hline 32 & 0.1041 & 0.00482 & 0.4693 & $2.63741 \mathrm{E}-4$ & $5.19332 \mathrm{E}-4$ & $9.7771 \mathrm{E}-6$ & 0.06563 & 0.00472 \\
\hline
\end{tabular}

Table S7 Fitting parameter obtained from Cole-Cole for $\mathbf{2}$ in 1000 Oe field.

\begin{tabular}{|c|c|c|c|c|c|c|c|c|}
\hline $\begin{array}{l}T \\
(\mathbf{K})\end{array}$ & $\begin{array}{l}\chi_{\mathrm{s}}\left(\mathrm{cm}^{3}\right. \\
\left.\mathrm{mol}^{-1}\right)\end{array}$ & $\operatorname{dev}\left(\chi_{\mathrm{s}}\right)$ & $\begin{array}{l}\chi_{\mathrm{T}}\left(\mathrm{cm}^{3} \mathrm{~mol}\right. \\
\left.{ }^{-1}\right)\end{array}$ & $\operatorname{dev}\left(\chi_{\mathrm{T}}\right)$ & $\tau(\mathbf{s})$ & $\operatorname{dev}(\tau)$ & $\alpha$ & $\operatorname{dev}(\alpha)$ \\
\hline 12 & 0.05008 & 0.00158 & 1.11504 & 0.01148 & 0.55265 & 0.01053 & 0.16779 & 0.00593 \\
\hline 14 & 0.04687 & 0.00134 & 0.9242 & 0.00444 & 0.22846 & 0.00211 & 0.13885 & 0.00426 \\
\hline 16 & 0.04371 & 0.00147 & 0.79929 & 0.003 & 0.10984 & $9.05043 \mathrm{E}-4$ & 0.12311 & 0.00435 \\
\hline 18 & 0.04215 & 0.00122 & 0.70791 & 0.00179 & 0.0586 & $3.76153 \mathrm{E}-4$ & 0.11573 & 0.00352 \\
\hline 20 & 0.04042 & 0.00124 & 0.63547 & 0.00138 & 0.03283 & $2.07106 \mathrm{E}-4$ & 0.1089 & 0.00351 \\
\hline 22 & 0.04206 & 0.00129 & 0.577 & 0.00113 & 0.01954 & $1.25024 \mathrm{E}-4$ & 0.09931 & 0.00362 \\
\hline 24 & 0.04469 & 0.00147 & 0.52953 & 0.00101 & 0.01136 & $8.0203 \mathrm{E}-5$ & 0.09392 & 0.00401 \\
\hline 26 & 0.04505 & 0.00212 & 0.48828 & 0.00105 & 0.00606 & $5.82686 \mathrm{E}-5$ & 0.09563 & 0.00534 \\
\hline 28 & 0.0452 & 0.00201 & 0.45428 & $6.06757 \mathrm{E}-4$ & 0.00278 & $2.31218 \mathrm{E}-5$ & 0.09775 & 0.0042 \\
\hline 30 & 0.05097 & 0.00417 & 0.42418 & $5.83638 \mathrm{E}-4$ & 0.00116 & $1.98611 \mathrm{E}-5$ & 0.09365 & 0.00639 \\
\hline 32 & 0.05081 & 0.01198 & 0.39709 & $5.18418 \mathrm{E}-4$ & 4.66018E-4 & $2.37421 \mathrm{E}-5$ & 0.0855 & 0.01085 \\
\hline
\end{tabular}


Table S8 Fitting parameters obtained from data of $\ln (\tau) v s \mathrm{~T}^{-1}$ for $\mathbf{1}$ and $\mathbf{2}$, containing Orbach, Raman or QTM processes.

\begin{tabular}{|lllll|}
\hline & $\mathbf{1}(0 \mathrm{Oe})$ & $\mathbf{1}(1000 \mathrm{Oe})$ & $\mathbf{2}(0 \mathrm{Oe})$ & $\mathbf{2}(1000 \mathrm{Oe})$ \\
\hline$\tau_{\text {QTM }}(\mathrm{s})$ & $9.8 \times 10^{-3}$ & - & $2.1 \times 10^{-1}$ & - \\
$C$ & $7.0 \times 10^{-1}$ & $1.3 \times 10^{-3}$ & $1.9 \times 10^{-2}$ & $2.6 \times 10^{-5}$ \\
$\mathrm{n}$ & 2.3 & 3.9 & 2.6 & 4.6 \\
$\tau_{0}(\mathrm{~s})$ & $5.5 \times 10^{-7}$ & $5.3 \times 10^{-6}($ fixed $)$ & $4.0 \times 10^{-9}$ & $7.9 \times 10^{-9}($ fixed $)$ \\
$c(\mathrm{~K})$ & 160 & $110($ fixed $)$ & 381 & $355($ fixed $)$ \\
\hline
\end{tabular}

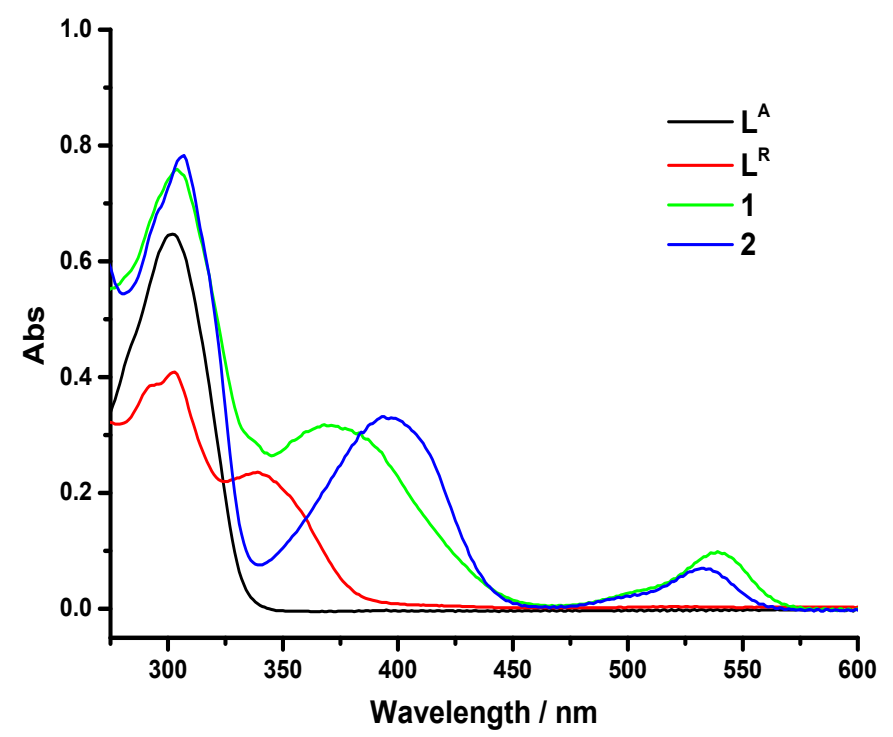

Figure S1. UV-vis spectra for $\mathrm{L}^{\mathrm{A}}, \mathrm{L}^{\mathrm{R}}$, complexes $\mathbf{1}$ and $\mathbf{2}$ in EtOH. 


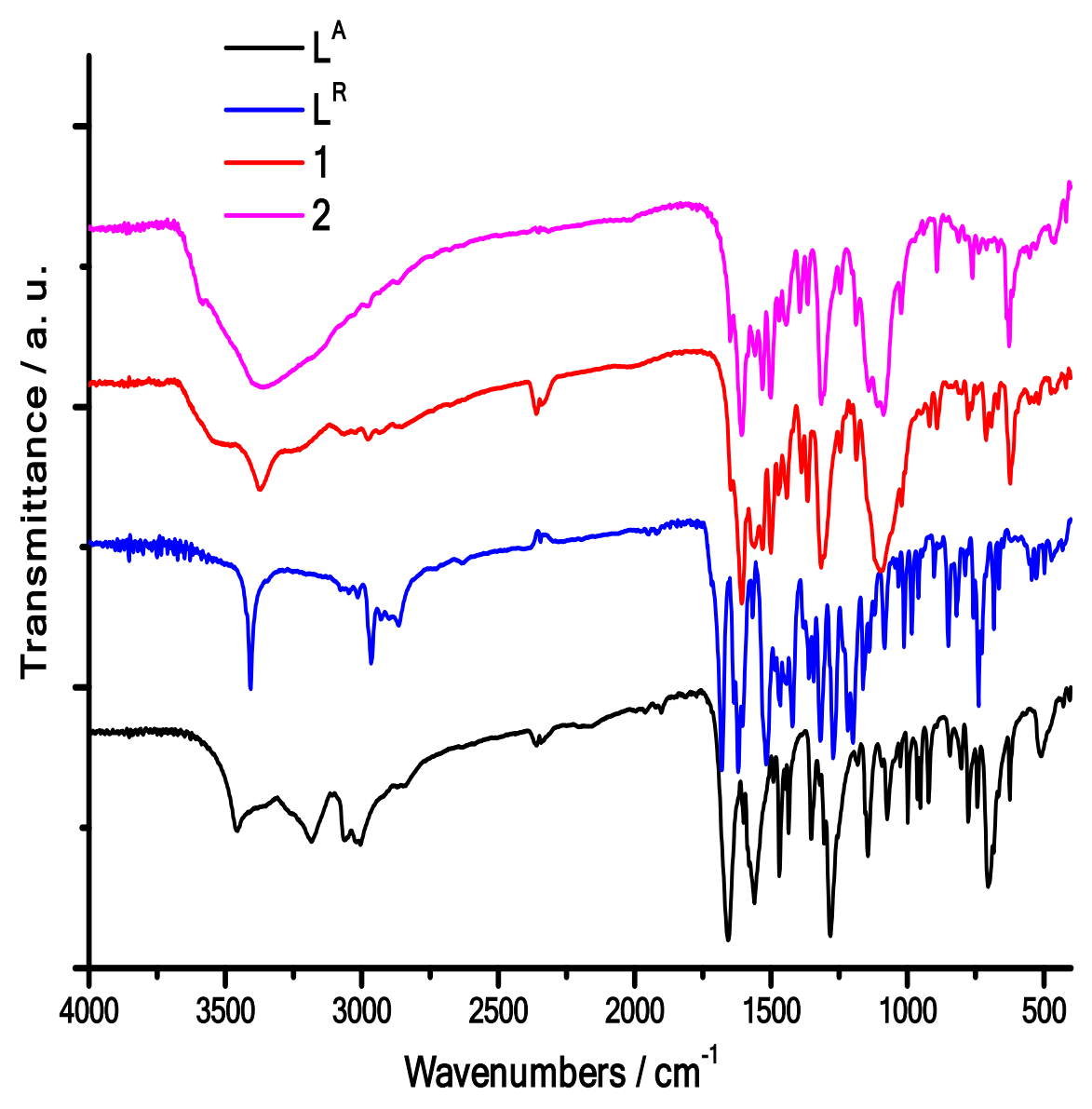

Figure S2. The IR spectra for $\mathrm{L}^{\mathrm{A}}, \mathrm{L}^{\mathrm{R}}$, complexes $\mathbf{1}$ and $\mathbf{2}$ with $\mathrm{KBr}$ tablets. 

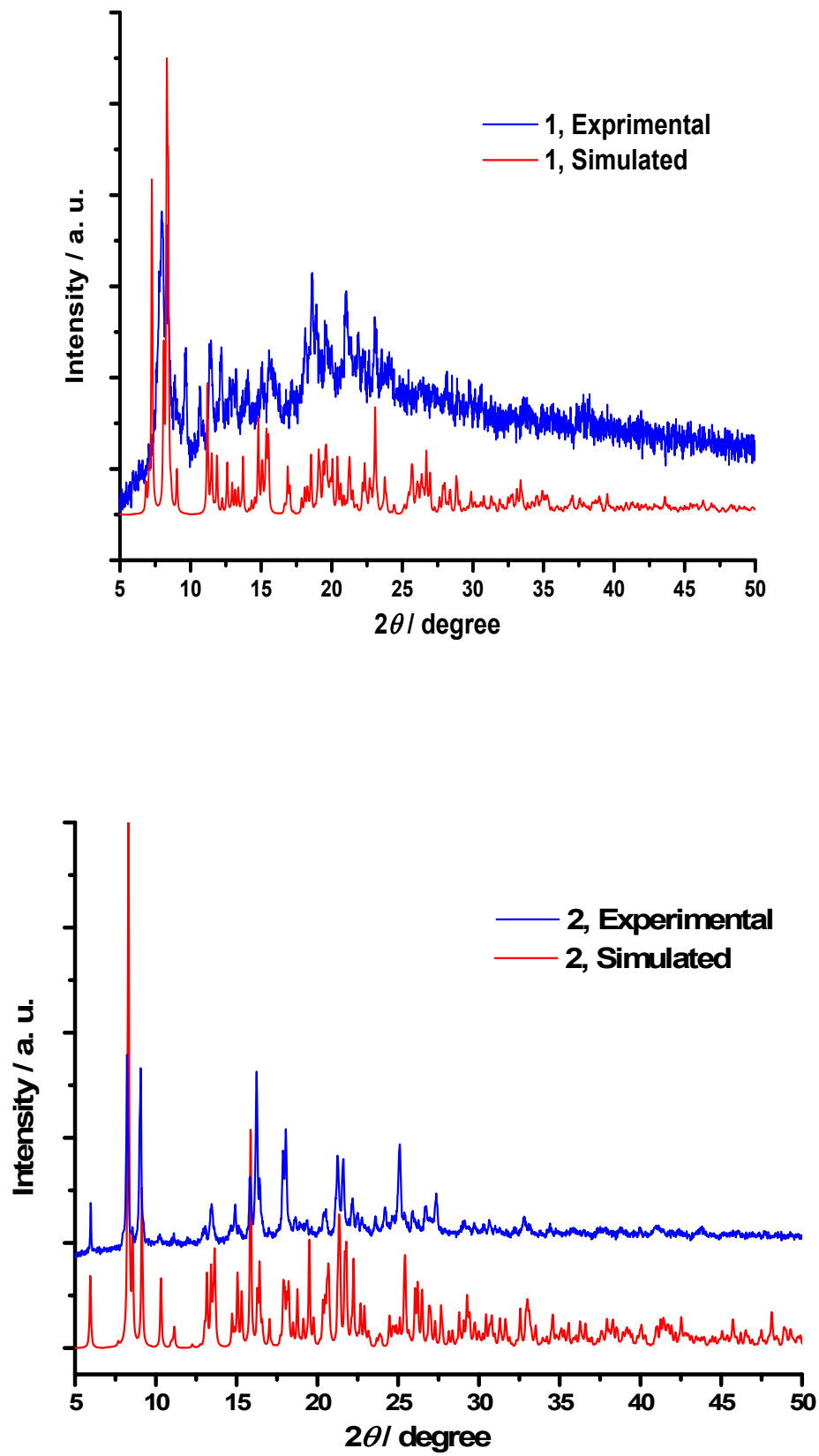

Figure S3. The powdered XRD pattern for complexes 1 (top) and 2 (bottom). 

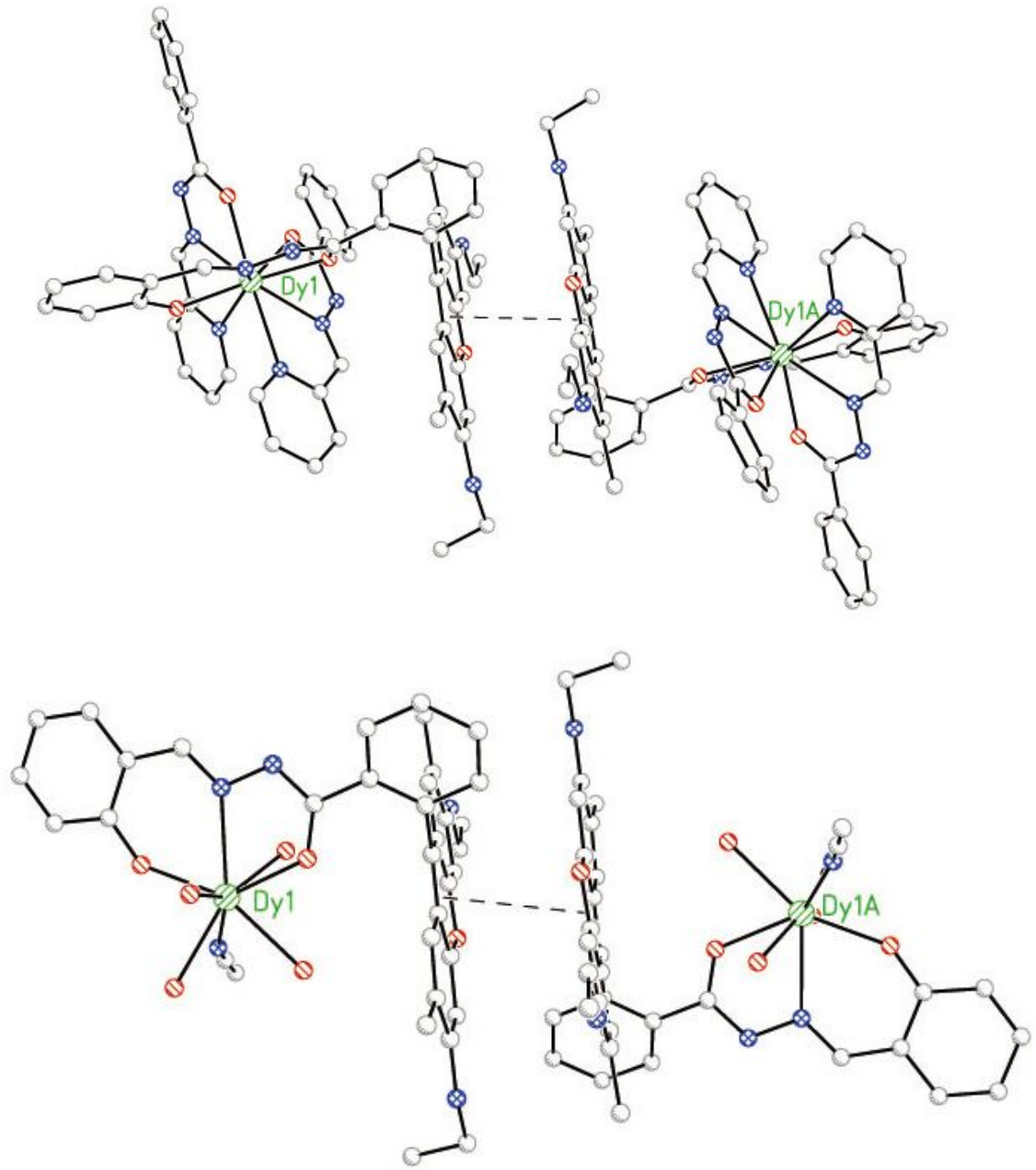

Figure S4. The intermolecular $\pi---\pi$ stacking for complexes 1 (top) and 2 (bottom). 

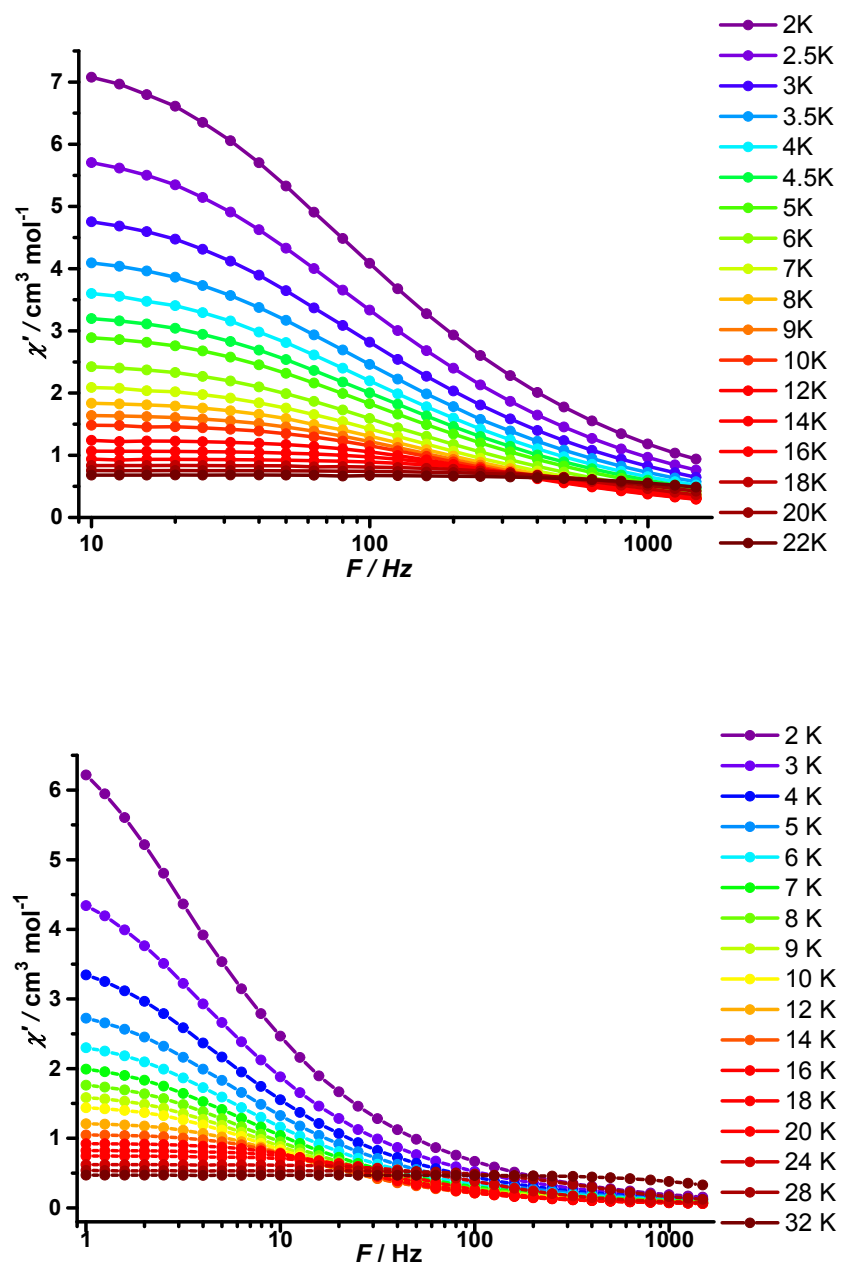

Figure S5. Frequency-dependence of the in-phase $(\chi$ ') ac susceptibilities for 1 (top) and for 2 (bottom) in zero dc field. 

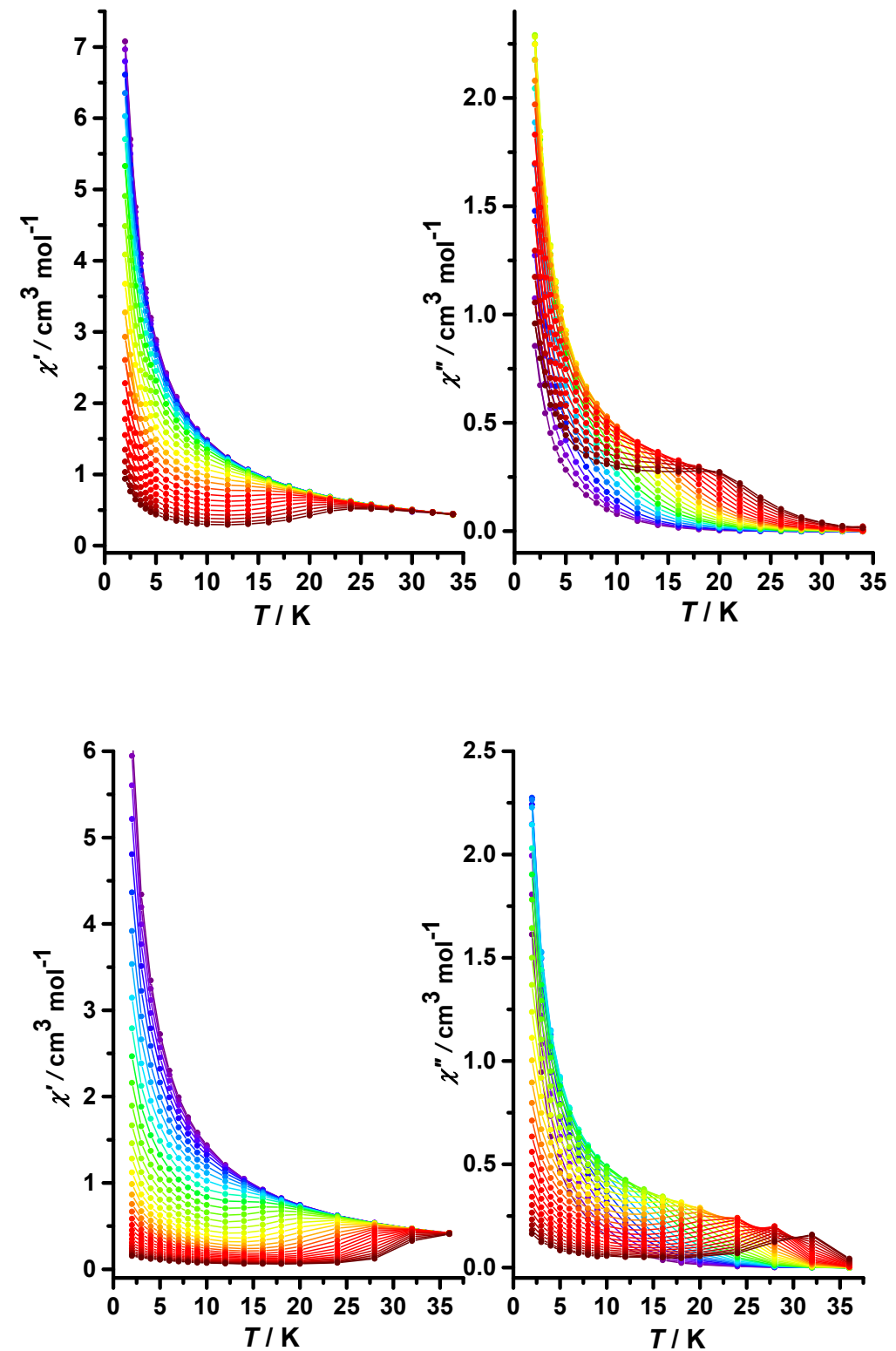

Figure S6. Temperature dependence of the in-phase $\left(\chi^{\prime}\right)$ and out-of-phase $(\chi$ ”) ac susceptibilities in zero dc field for 1 from 1488 to $10 \mathrm{~Hz}$ (top) and for 2 from 1488 to 1 $\mathrm{Hz}$ (bottom), respectively. 

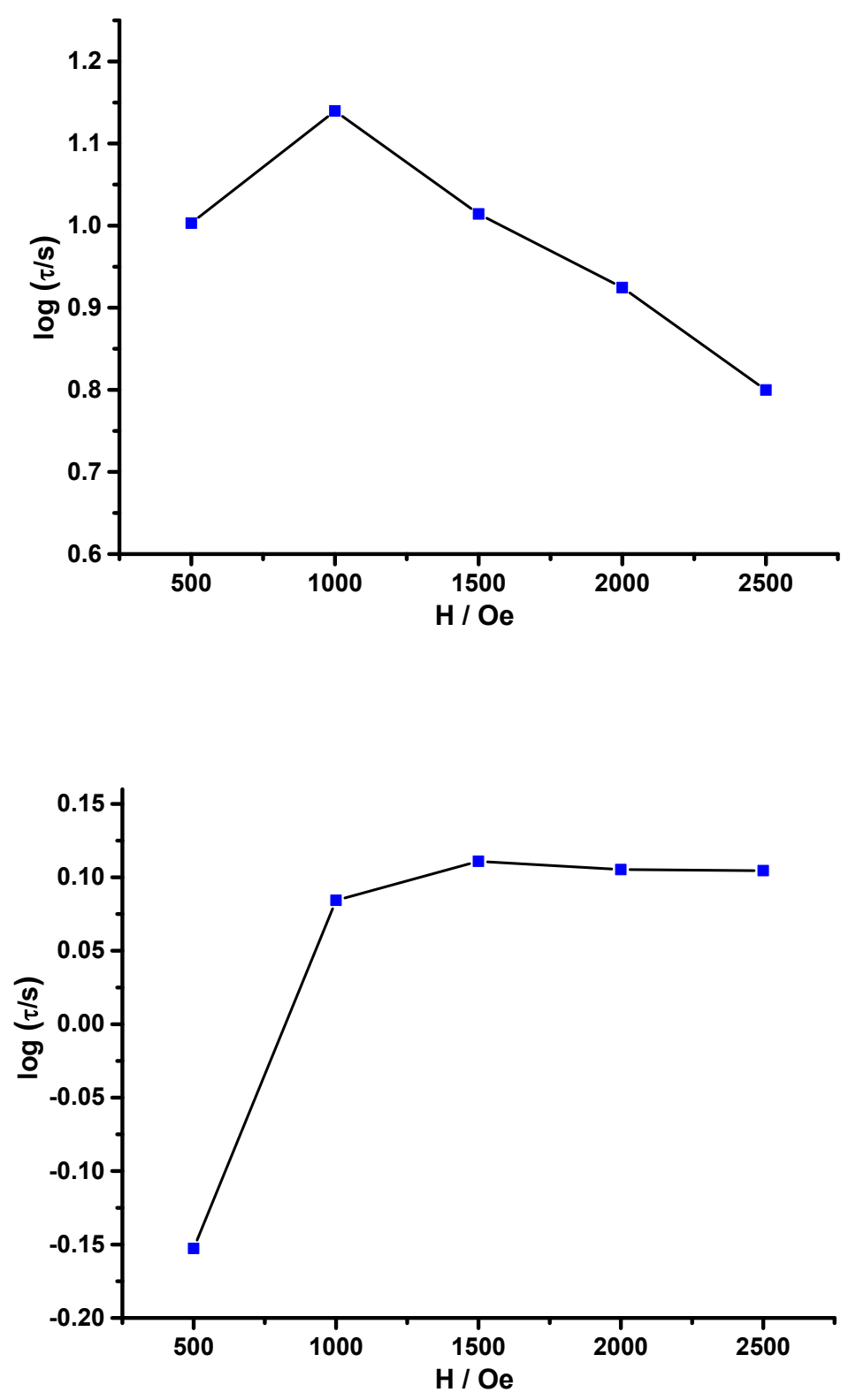

Figure S7. Field dependence of relaxation times for $\mathbf{1}$ at $4 \mathrm{~K}$ (top) and for $\mathbf{2}$ at $10 \mathrm{~K}$ (bottom), respectively. 

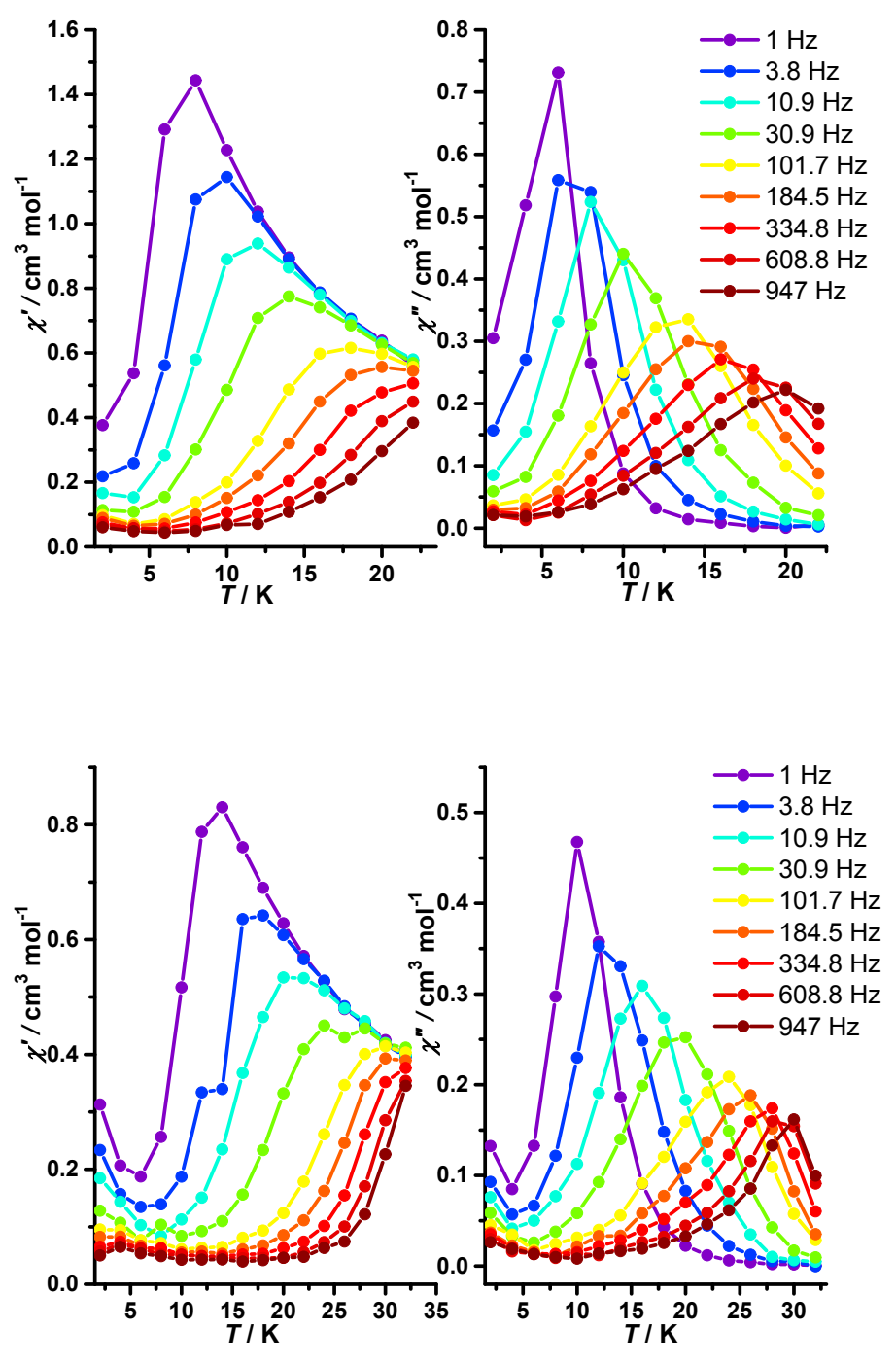

Figure S8. Temperature dependence of the in-phase $\left(\chi^{\prime}\right)$ and out-of-phase $(\chi$ ”) ac susceptibilities in 1000 Oe dc field for $\mathbf{1}$ (top) and for $\mathbf{2}$ (bottom), respectively. 
(a)

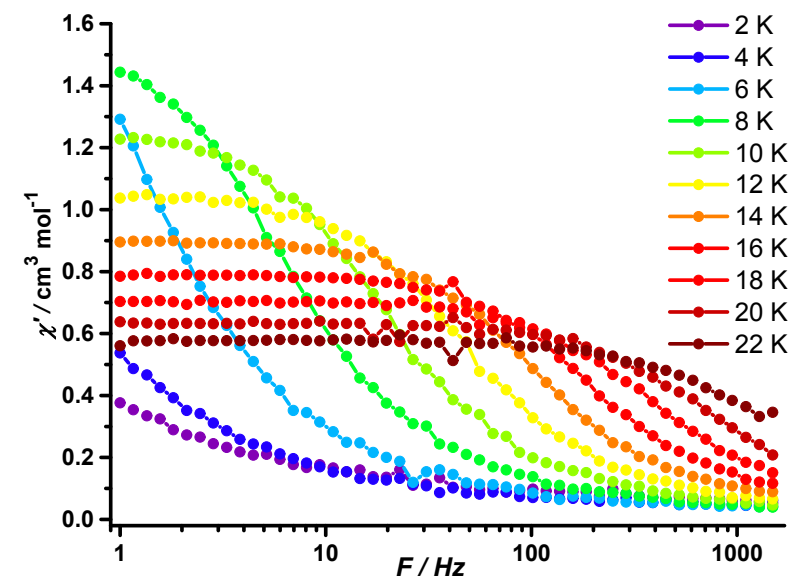

(b)

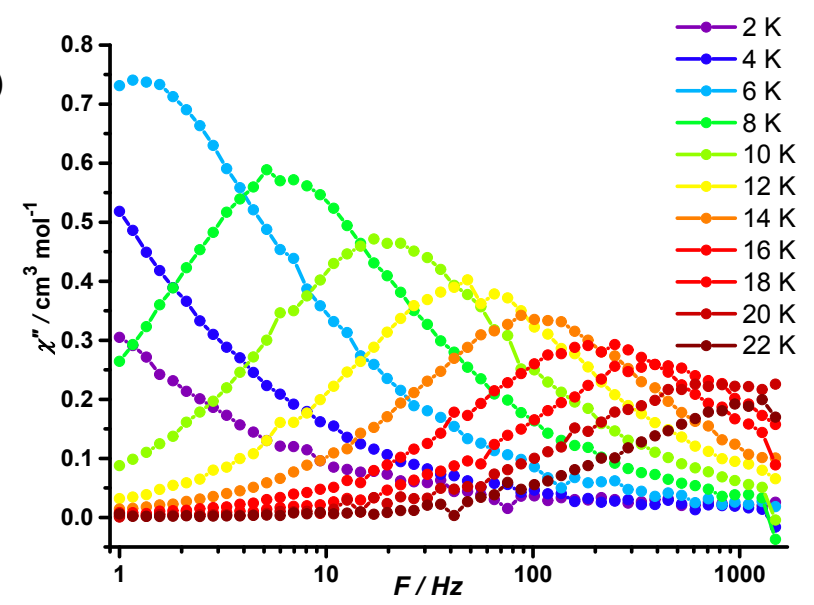

(c)

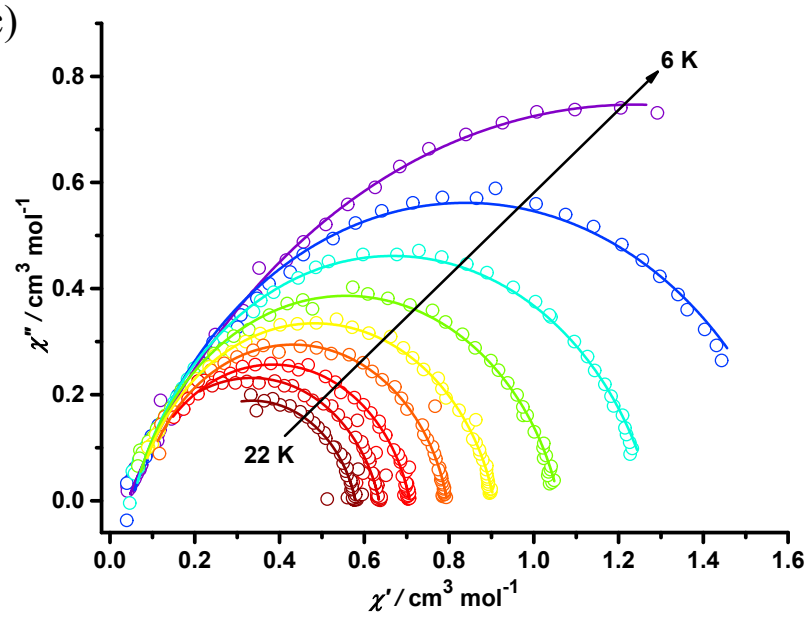

Figure S9. Frequency-dependence of the in-phase (a), out-of-phase (b) ac susceptibilities and Cole-Cole data (c) for $\mathbf{1}$ in 1000 Oe dc field. 
(a)

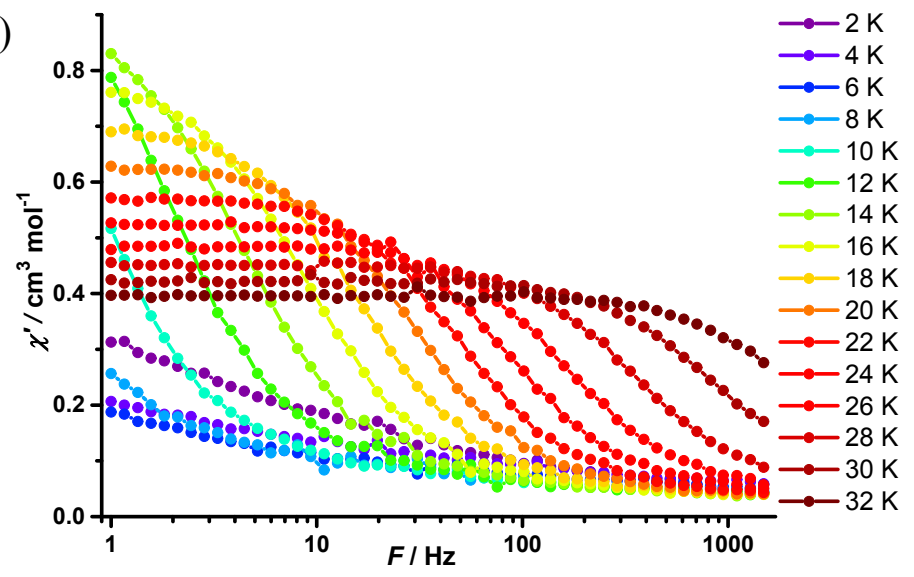

(b)
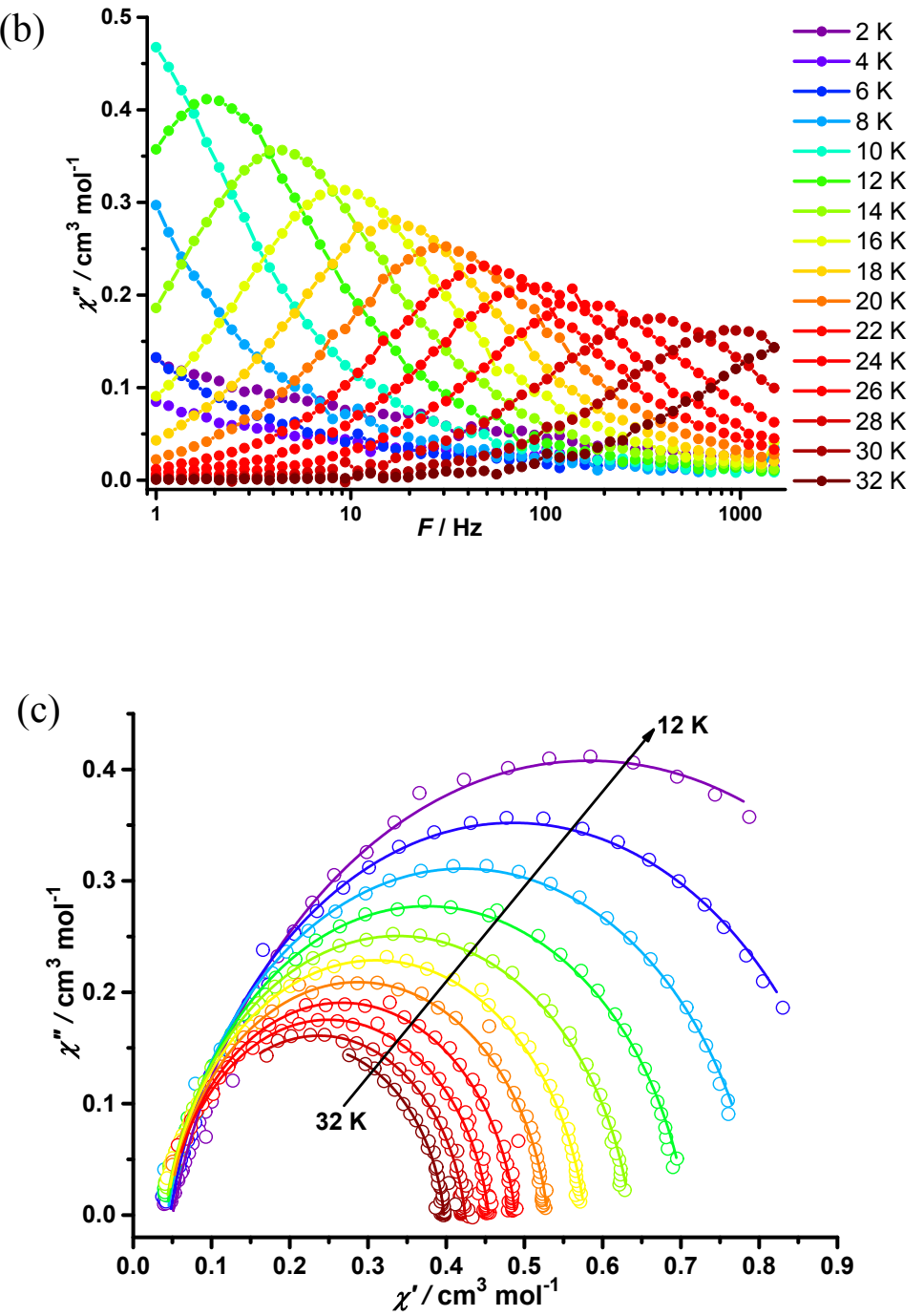

Figure S10. Frequency-dependence of the in-phase (a), out-of-phase (b) ac susceptibilities and Cole-Cole data (c) for $\mathbf{2}$ in 1000 Oe dc field. 


\section{Computational details}

Complete-active-space self-consistent field (CASSCF) calculations on complexes $\mathbf{1}$ and $\mathbf{2}$ (see Figure S11 for the calculated complete molecular structures) on the basis of single-crystal X-ray determined geometries have been carried out with MOLCAS 8.2 program package. ${ }^{\mathrm{S} 1}$

The basis sets for all atoms are atomic natural orbitals from the MOLCAS ANO-RCC library: ANO-RCC-VTZP for Dy ${ }^{I I I}$ ion; VTZ for close O and N; VDZ for distant atoms. The calculations employed the second order Douglas-Kroll-Hess Hamiltonian, where scalar relativistic contractions were taken into account in the basis set and the spin-orbit couplings were handled separately in the restricted active space state interaction (RASSI-SO) procedure. For both complexes, active electrons in 7 active spaces include all $f$ electrons $(\operatorname{CAS}(9$ in 7$))$ in the CASSCF calculation. To exclude all the doubts, we calculated all the roots in the active space. We have mixed the maximum number of spin-free state which was possible with our hardware (all from 21 sextets, 128 from 224 quadruplets, 130 from 490 doublets). SINGLE_ANISO ${ }^{\mathrm{S} 2}$ program was used to obtain the energy levels, $\boldsymbol{g}$ tensors, $m_{J}$ values, magnetic axes, magnetic susceptibilities et al., based on the above CASSCF/RASSI-SO calculations.
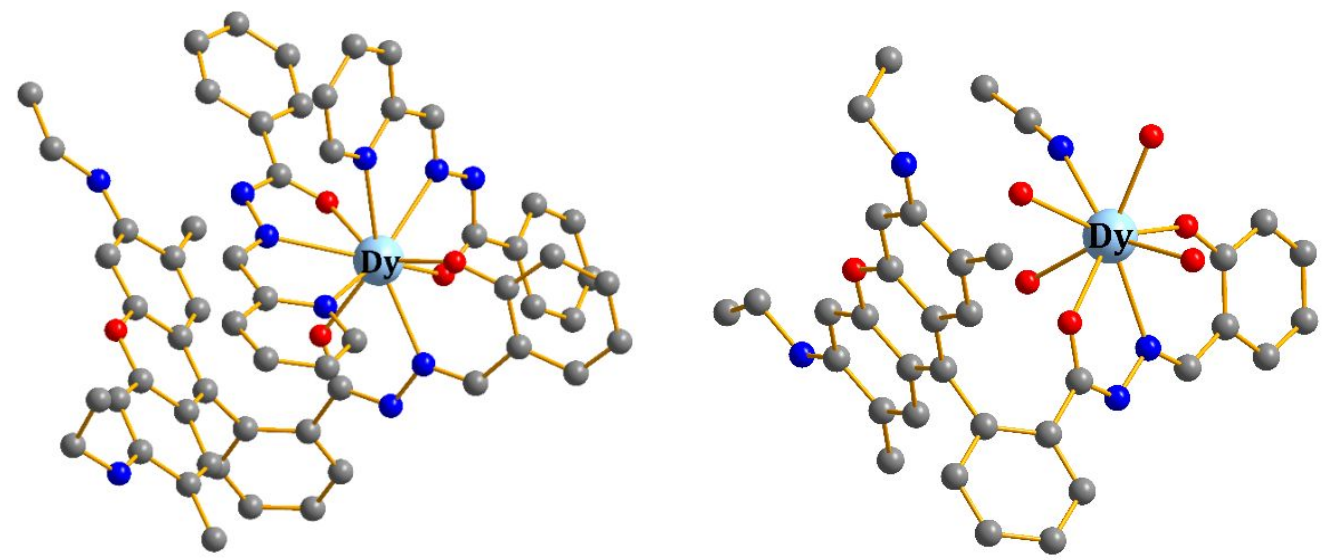

Figure S11. Calculated complete molecular structures of complexes 1 (left) and 2 (right), respectively. $\mathrm{H}$ atoms are omitted. 
Table S9 Calculated energy levels $\left(\mathrm{cm}^{-1}\right), \boldsymbol{g}\left(g_{\mathrm{x}}, g_{\mathrm{y}}, g_{\mathrm{z}}\right)$ tensors and predominant $m_{J}$ values of the lowest eight Kramers doublets (KDs) of individual Dy ${ }^{\mathrm{III}}$ fragments in complexes 1 and 2 using CASSCF/RASSI-SO with MOLCAS 8.2.

\begin{tabular}{|c|c|c|c|c|c|c|}
\hline \multirow{2}{*}{ KDs } & \multicolumn{3}{|c|}{1} & \multicolumn{3}{|c|}{2} \\
\hline & $E$ & $g$ & $m_{J}$ & $E$ & $g$ & $m_{J}$ \\
\hline 1 & 0.0 & $\begin{array}{c}0.005 \\
0.008 \\
19.699\end{array}$ & $\pm 15 / 2$ & 0.0 & $\begin{array}{c}0.001 \\
0.003 \\
19.679\end{array}$ & $\pm 15 / 2$ \\
\hline 2 & 221.3 & $\begin{array}{c}0.123 \\
0.164 \\
16.773\end{array}$ & $\pm 13 / 2$ & 223.1 & $\begin{array}{c}0.496 \\
1.295 \\
15.838\end{array}$ & $\pm 13 / 2$ \\
\hline 3 & 368.5 & $\begin{array}{c}1.453 \\
1.913 \\
12.913\end{array}$ & $\pm 11 / 2$ & 257.3 & $\begin{array}{c}0.287 \\
1.287 \\
17.465\end{array}$ & $\pm 7 / 2$ \\
\hline 4 & 423.2 & $\begin{array}{l}9.994 \\
6.686 \\
3.305\end{array}$ & $\pm 9 / 2$ & 358.1 & $\begin{array}{c}3.144 \\
4.134 \\
10.697\end{array}$ & $\pm 11 / 2$ \\
\hline 5 & 484.4 & $\begin{array}{c}1.090 \\
5.363 \\
10.423\end{array}$ & $\pm 7 / 2$ & 432.9 & $\begin{array}{l}9.248 \\
5.635 \\
1.814\end{array}$ & $\pm 3 / 2$ \\
\hline 6 & 535.6 & $\begin{array}{c}1.291 \\
5.415 \\
10.036\end{array}$ & $\pm 3 / 2$ & 492.2 & $\begin{array}{c}2.511 \\
2.845 \\
14.926\end{array}$ & $\pm 1 / 2$ \\
\hline 7 & 583.0 & $\begin{array}{c}1.159 \\
2.876 \\
14.557\end{array}$ & $\pm 1 / 2$ & 529.8 & $\begin{array}{c}0.323 \\
0.430 \\
19.183\end{array}$ & $\pm 5 / 2$ \\
\hline 8 & 638.8 & $\begin{array}{c}0.389 \\
1.074 \\
18.461\end{array}$ & $\pm 5 / 2$ & 721.6 & $\begin{array}{c}0.016 \\
0.027 \\
19.721\end{array}$ & $\pm 9 / 2$ \\
\hline
\end{tabular}

Table S10. Wave functions with definite projection of the total moment $\mid m_{J}>$ for the lowest two KDs for complexes $\mathbf{1}$ and $\mathbf{2}$.

\begin{tabular}{|c|c|c|}
\hline & $E$ & wave functions \\
\hline \multirow{3}{*}{1} & 0.0 & $97.32 \% \mid \pm 15 / 2>$ \\
\cline { 2 - 3 } & 221.3 & $94.92 \%| \pm 13 / 2>+3.17 \%| \pm 9 / 2>$ \\
\hline \multirow{2}{*}{2} & 0.0 & $96.76 \% \mid \pm 15 / 2>$ \\
\cline { 2 - 3 } & 223.1 & $78.54 \%|+13 / 2>+15.67 \%| \pm 9 / 2>$ \\
\hline
\end{tabular}




\section{References:}

S1 Aquilante, F.; Autschbach, J.; Carlson, R. K.; Chibotaru, L. F.; Delcey, M. G.; De Vico, L.; Galván, I. Fdez.; Ferré, N.; Frutos, L. M.; Gagliardi, L.; Garavelli, M.; Giussani, A.; Hoyer, C. E.; Li Manni, G.; Lischka, H.; Ma, D.; Malmqvist, P. Å.; Müller, T.; Nenov, A.; Olivucci, M.; Pedersen, T. B.; Peng, D.; Plasser, F.; Pritchard, B.; Reiher, M.; Rivalta, I.; Schapiro, I.; Segarra-Martí, J.; Stenrup, M.; Truhlar, D. G.; Ungur, L.; Valentini, A.; Vancoillie, S.; Veryazov, V.; Vysotskiy, V. P.; Weingart, O.; Zapata, F.; Lindh, R. MOLCAS 8: New Capabilities for Multiconfigurational Quantum Chemical Calculations across the Periodic Table, J. Comput. Chem., 2016, 37, 506.

S2 (a) Chibotaru, L. F.; Ungur, L.; Soncini, A. The Origin of Nonmagnetic Kramers Doublets in the Ground State of Dysprosium Triangles: Evidence for a Toroidal Magnetic Moment. Angew. Chem. Int. Ed., 2008, 47, 4126. (b) Ungur, L.; Van den Heuvel, W.; Chibotaru, L. F. Ab initio investigation of the non-collinear magnetic structure and the lowest magnetic excitations in dysprosium triangles. New J. Chem., 2009, 33, 1224. (c) Chibotaru, L. F.; Ungur, L.; Aronica, C.; Elmoll, H.; Pilet, G.; Luneau, D. Structure, Magnetism, and Theoretical Study of a Mixed-Valence $\mathrm{Co}^{\mathrm{II}}{ }_{3} \mathrm{Co}^{\mathrm{III}}{ }_{4}$ Heptanuclear Wheel: Lack of SMM Behavior despite Negative Magnetic Anisotropy. J. Am. Chem. Soc., 2008, 130, 12445. 